\title{
Optical properties of analogs of Titan's aerosols produced by dusty plasma
}

\author{
E. Hadamcik ${ }^{1}$, J.-B. Renard ${ }^{2}$, A. Mahjoub ${ }^{1}$, T. Gautier ${ }^{1}$, N. Carrasco ${ }^{1}$, G. Cernogora ${ }^{1}$, and C. Szopa ${ }^{1}$ \\ ${ }^{1}$ UPMC (U.P. \& M. Curie, Sorbonne Universités), Université Versailles St-Quentin, CNRS/INSU, LATMOS-IPSL, Paris, France \\ ${ }^{2}$ LPC2E, CNRS, Orléans, France
}

(Received October 31, 2012; Revised May 24, 2013; Accepted May 30, 2013; Online published October 24, 2013)

\begin{abstract}
Analogs of Titan's aerosols are produced in the laboratory as grains in a gas mixture, or as layers on a substrate. This production procedure enables the methane-nitrogen mixture composition to be changed. The aim of this paper is to understand the variations observed on the linear polarization of the scattered light as a function of the production conditions. The influence of the concentration of methane injected in the plasma will be discussed and compared with the previous work of Hadamcik et al. (2009a). The diameter of the grains are measured by SEMFEG images. The decrease of absorption with increasing wavelength, measured by spectroscopic ellipsometry on layers, is observed for a decreasing initial methane ratio and analyzed in terms of an increasing 'amine' content in the materials. The phase function parameters of the linear polarization of the scattered light are discussed in terms of the diameters of the aggregates and of the constituent grains, and the variation of the refractive indices (mainly absorption). The polarization is found to be highly correlated with the constituent grain size. Finally, the experimental results are compared to polarization measurements from space of the Titan's atmosphere.
\end{abstract}

Key words: Light scattering, polarization, refractive indices, tholins, Titan, aerosol.

\section{Introduction}

Titan's atmosphere is mainly composed of nitrogen $\mathrm{N}_{2}$ and methane $\mathrm{CH}_{4}$. The $\mathrm{CH}_{4} / \mathrm{N}_{2}$ ratio varies with altitude. Energetic particles from Saturn's magnetosphere and photochemical reactions dissociate $\mathrm{N}_{2}$ and $\mathrm{CH}_{4}$. The subsequent chemistry produces solid aerosols in the upper atmosphere which are widespread all around Titan through the atmospheric general circulation, giving the characteristic yellow color to the satellite.

Some physical properties of these aerosols have been deduced from the solar light scattered by the particles and its linear polarization. The observations have been performed by different space probes: Pioneer 11, Voyager 2 and Huygens (Tomasko and Smith, 1982; West et al., 1983; Tomasko et al., 2005). From a comparison between observations and numerical simulations of the particles and the light they scatter, fractal cluster-cluster aggregates with 3000 to 4500 constituent grains of $80-\mathrm{nm}$ mean diameter seem to best describe Titan's aerosols structure (Tomasko et al., 2008, 2009). The input parameters for the simulations (e.g. refractive indices) are obtained by measurements on analogs called tholins, which are produced in the laboratory by photochemical reactions or plasma discharges (Khare et al., 1984; Imanaka et al., 2004; Mahjoub et al., 2012; Sciamma-O'Brien et al., 2012).

The aim of this paper is to study the influence of the methane content of the plasma discharge on the linear polarization of the light scattered by tholins produced by the PAMPRE set-up (described in detail in Szopa et al., 2006).

Copyright (C) The Society of Geomagnetism and Earth, Planetary and Space Sciences (SGEPSS); The Seismological Society of Japan; The Volcanological Society of Japan; The Geodetic Society of Japan; The Japanese Society for Planetary Sciences; TERRAPUB.

doi:10.5047/eps.2013.05.019
This work completes an initial study carried out by Hadamcik et al. (2009a) on this type of tholins. The experiment produces tholins in the gas phase as dust particles, or as thin films on substrates. The linear polarization $P$ of the light scattered by the dust particles is measured with the PROGRA $^{2}$ instrument (see Renard et al., 2002, for further description). $P$ depends on the geometry of observations, which is expressed by the phase angle $\alpha$ (the angle between the direction of the incident light and the line of sight of the detector, as seen from the dust particle), on the morphology and size distribution of the particles. $P$ also depends on the light wavelength, $\lambda$, by the size parameter dependence ( $X=\pi d / \lambda, d$ is the diameter). It was first defined for spherical particles in the Mie theory. For irregular particles, an effective diameter is defined as the diameter of the sphere with an equivalent volume. The real and imaginary refractive indices $n(\lambda)$ and $k(\lambda)$, reflect the chemical composition of the materials. In Hadamcik et al. (2009a), the influence of $k$ on scattered light polarization was tentatively interpreted through $k(\lambda)$ values in the visible range taken from the literature with tholins other than those produced by the PAMPRE set-up.

Since Hadamcik et al. (2009a), new samples have been produced allowing the variations of the tholins' properties to be characterized more precisely, as a function of the injected methane percentage (hereafter, $\mathrm{CH}_{4}$ ratio), such as the sizes and size distributions of the particles. Furthermore, the refractive indices in the 340-1000 nm range were measured on proper PAMPRE samples (Mahjoub et al., 2012), enabling the polarization results to be interpreted. The evolution of the chemical composition of the material according to the initial methane amount was studied by infrared absorption spectroscopy giving an insight into the 


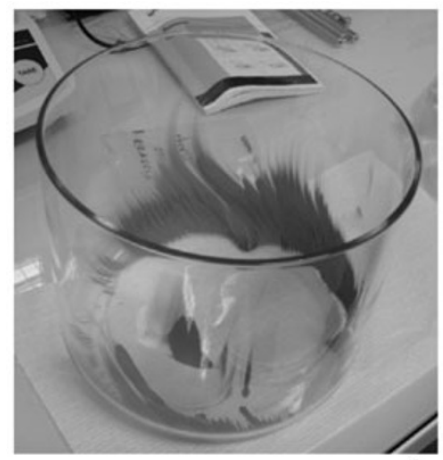

Glass vessel a With sticked tholins

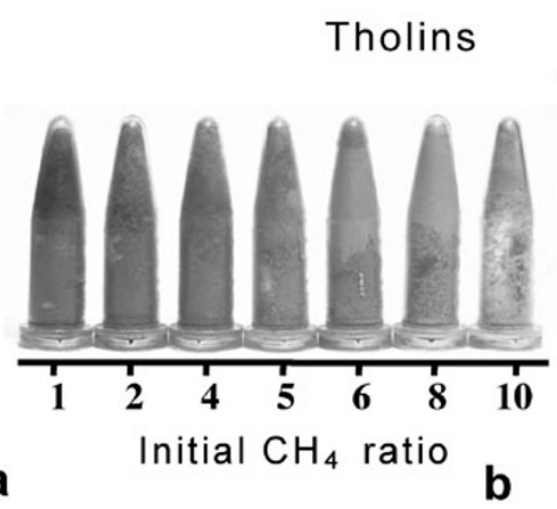

b

Fig. 1. Tholins produced by the PAMPRE instrument. (a) Glass vessel coated by tholins (b). Vials filled with different tholins showing a clearer brown color for the tholins when the $\mathrm{CH}_{4}$ ratio increases. The change from dark to clear may be the result of larger grains, or of a lower absorption of the bulk materials in the visible range.

change in albedo of the materials. Finally, the variation of the linear polarization, $P$, as a function of the different characteristics of tholins, is presented, and $P$ is compared with the results obtained by the different space missions.

\section{Production of Tholins by the PAMPRE Set-Up}

Tholins are produced in the PAMPRE experiment, a CCP (Capacitively Coupled Plasma) radio frequency discharge at 13.6 $\mathrm{MHz}$ in a $\mathrm{N}_{2}$ and $\mathrm{CH}_{4}$ gas mixture, which is described in detail in Szopa et al. (2006) and Sciamma-O'Brien et al. (2010). The pressure is $0.9 \mathrm{hPa}$ for all the experimental conditions presented here.

The discharge induces the formation of ions, atoms and radicals by the dissociation of methane $\mathrm{CH}_{4}$ and nitrogen $\mathrm{N}_{2}$ molecules by electronic collisions. These reactive species recombine, forming heavy gas species and tholins. The discharge can work in CWC (Continuous Working Conditions) for hours, or in a pulsed mode in order to produce tholins with a different reduced discharge duration.

The plasma is confined by a metallic grid and surrounded by a glass vessel where the tholins are collected and finally stored in microvials (Fig. 1). For film deposition, the substrates are placed on the grounded electrode. The samples are analyzed ex-situ. Films are used to measure the refractive indices and to characterize the materials composition by infrared spectroscopy. The light scattering is studied on tholins' grains. According to Quirico et al. (2008), the materials in layers and tholins in the present work are considered to be made of the same materials. Indeed, similar patterns were found in the mid-infrared spectrum for films and thin layers of tholins produced in the PAMPRE set-up, with $1 \%, 2 \%$ and $10 \%$ initial $\mathrm{CH}_{4}$ ratios. The $\mathrm{CH}_{4} / \mathrm{N}_{2}$ ratio between $1 \%$ and $10 \%$ influence is emphasised in the present work.

\section{Morphology and Size of the Dust Grains (SEM Analysis)}

Hadamcik et al. (2009a) have presented a first study of the morphology of the grains obtained from SEM-FEG images. The grains are quasi-spherical. Their surface is not completely smooth. Some grains are broken and show radial structures corresponding to the surface irregularities, which fill up each grain suggesting that the grains grow radially. Some aggregates appear on the images, with just touching grains or with sintered ones. The aggregation may occur in the discharge or when the grains stick to the glass vessel or inside the microvials (Fig. 1). In the present paper, the word 'grain' is used for the individual grains (often called monomers in other papers) and the association of grains are referred to as particles or aggregates.

For each sample, numerous images are taken and the size distribution of the grains are measured. In the following, the size is defined as the diameter of the grains. Several thousand grains are necessary to obtain a good statistics. The size distributions are fitted by a Gaussian function, the size at maximum is called the average size and the half-width at half-maximum is indicated. The average size and size distribution depends on the pulse duration (Fig. 2) and on the $\mathrm{CH}_{4}$ ratio (Fig. 3). The average size increases with the increase of the plasma duration up to a steadystate maximum, depending on the $\mathrm{CH}_{4}$ ratio (Fig. 2). For durations larger than tens of seconds, the size distribution does not change anymore and reaches the CWC: $150 \mathrm{~s}$ for $2 \% \mathrm{CH}_{4}$ ratio and $300 \mathrm{~s}$ for $10 \% \mathrm{CH}_{4}$ ratio. The average size in CWC is plotted as a function of $\mathrm{CH}_{4}$ ratios (Fig. 3). It can be fitted by a polynomial function, and presents a minimum for $4-5 \% \mathrm{CH}_{4}$ ratios.

\section{Refractive Indices Measurements}

The light scattering experiments are performed at two wavelengths: green light at $543.5 \mathrm{~nm}(\mathrm{G})$ and red light at $632.8 \mathrm{~nm}(\mathrm{R})$ (see below Sections 6.1 and 6.6). The optical indices, $n$ and $k$, of the various samples are therefore needed for these two wavelengths.

The variation of the optical indices as a function of the methane amount has been published in Mahjoub et al. (2012). As is also shown in Sciamma-O'Brien et al. (2012), $n$ and $k$ decrease when the wavelength increases: the $n$ and $k$ values are expected to be smaller for the red light. Figure 4 presents the extracted values of the refractive indices, 


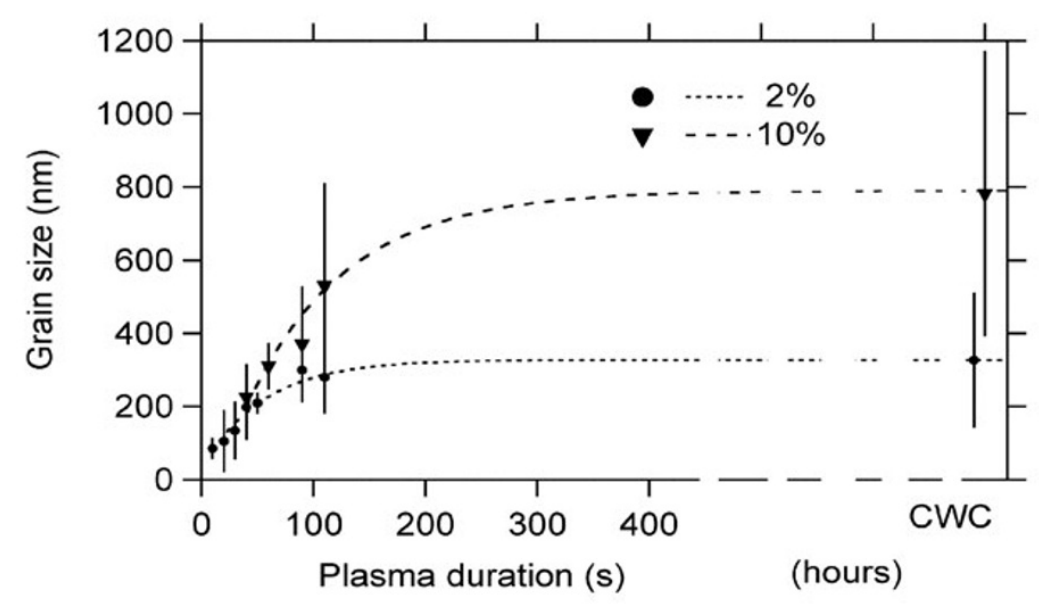

Fig. 2. Comparison of the average size variation vs plasma duration between $2 \%$ and $10 \%$ ratios. Error bars are the half width of the Gaussian size distribution fits.

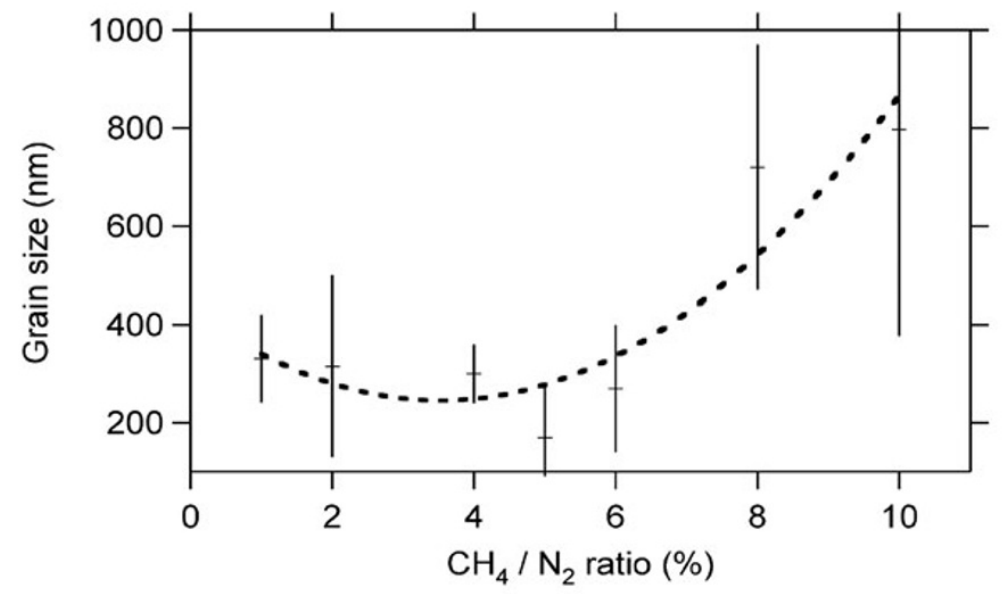

Fig. 3. Average size of tholins with different $\mathrm{CH}_{4}$ ratios produced in $\mathrm{CWC}$ conditions. Error bars are the half width of the Gaussian size distribution fits.
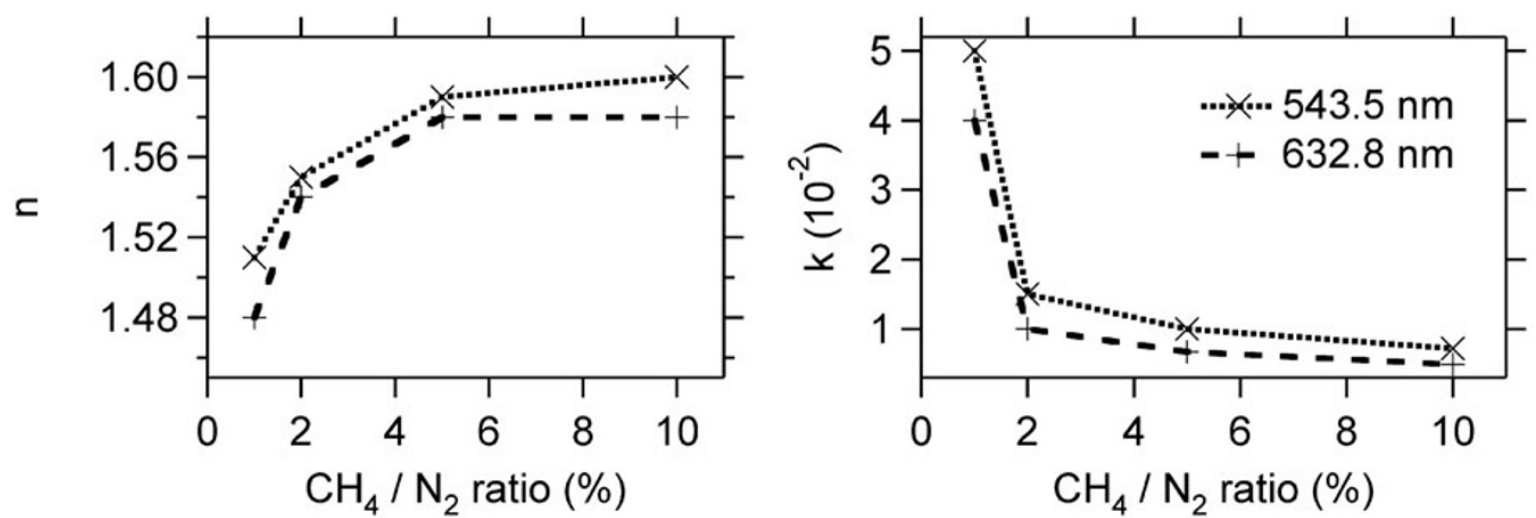

Fig. 4. Variations of $n(\lambda)$ and $k(\lambda)$ as a function of the $\mathrm{CH}_{4}$ ratio for $543.5 \mathrm{~nm}$ and $632.8 \mathrm{~nm}$.

$n$ and $k$, as a function of the $\mathrm{CH}_{4}$ ratio for both wavelengths. in the $1-2 \% \mathrm{CH}_{4}$ concentration range, and from $1 \times 10^{-2}$ to For both $G$ and $R, n$ increases significantly from about $1.5 \quad 5 \times 10^{-3}$ in the $2-10 \% \mathrm{CH}_{4}$ concentration range. The ratio to 1.6 with the $\mathrm{CH}_{4}$ ratio. The deviation between $n_{G}$ and $n_{R}$ is smaller than 0.02 for all $\mathrm{CH}_{4}$ ratios. The imaginary index $k$ varies drastically from about $5 \times 10^{-2}$ to about $1 \times 10^{-2}$ $k_{G} / k_{R}$ is slightly smaller for the $1 \% \mathrm{CH}_{4}$ sample $(\sim 1.25)$ than for the other concentrations $(\sim 1.5)$. The influence of these parameters on the light scattering and polarization are 


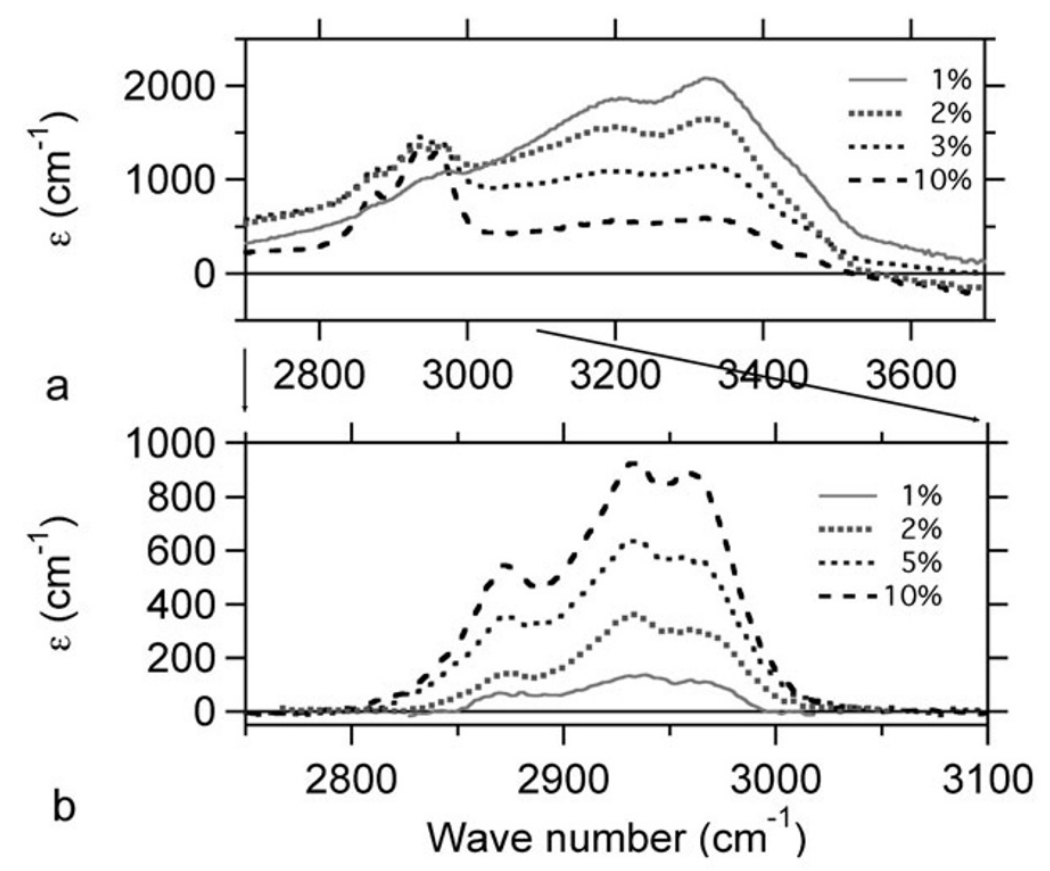

Fig. 5. Mid-infrared spectrum for different $\mathrm{CH}_{4}$ ratios. (a) $2700-3700 \mathrm{~cm}^{-1}$ wave number range. (b) Magnification of the $2700-3100 \mathrm{~cm}-1$ region after subtraction of the base-line. $\epsilon=$ near absorption coefficients.

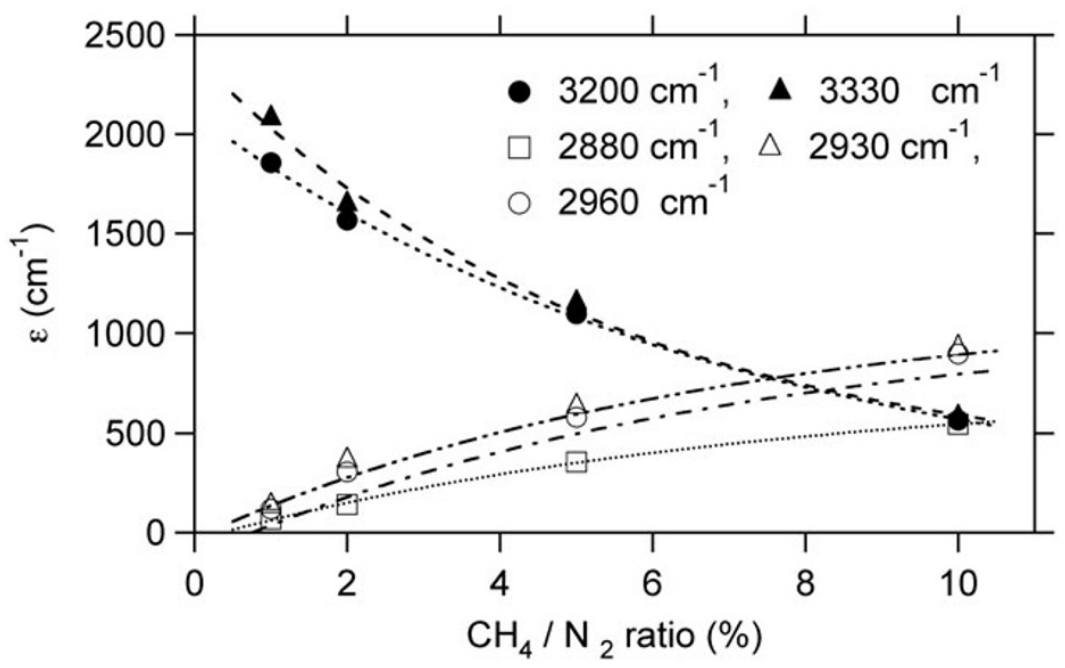

Fig. 6. Variations of $\epsilon=$ linear absorption coefficient for the main bands: 'amine' black markers and 'aliphatic' open markers.

correlated and depends also on the geometrical characteristics of the particles.

The chemical composition of tholins changes with the $\mathrm{CH}_{4}$ ratio, as shown by elemental analysis (SciammaO'Brien et al., 2010). This change can be a clue for understanding the evolution of the refractive indices with the $\mathrm{CH}_{4}$ ratio. Mid-infrared spectroscopy is therefore used to further characterize some molecular bands in the materials.

\section{Mid-Infrared Absorption Spectroscopy}

Mid-infrared absorption spectroscopy was used to partially interpret the variation of color and absorption in terms of chemical composition. The evolution of linear absorption coefficients $\epsilon$ (in $\mathrm{cm}^{-1}$, i.e. absorbance normalized per thickness-unit) in the range $2.8 \mu \mathrm{m}-4 \mu \mathrm{m}\left(3500 \mathrm{~cm}^{-1}\right.$
$2500 \mathrm{~cm}^{-1}$ ) for tholins samples produced at different $\mathrm{CH}_{4}$ ratios were studied in Gautier et al. (2012). In this spectral region, two groups of absorption bands are identified as being due to amine function- $-\mathrm{NH}_{2}$ and possibly- $\mathrm{NH}-$ $\mathrm{NH}, \mathrm{NH}_{2}$ (near $3200 \mathrm{~cm}^{-1}$ ). The aliphatic carbon bands$\mathrm{CH}_{2}$ - and $-\mathrm{CH}_{3}$ (between $2800 \mathrm{~cm}^{-1}$ and $2900 \mathrm{~cm}^{-1}$ ) are superimposed. These data are here further treated with a focus on the amine and aliphatic contributions. The linear absorption coefficients obtained for different $\mathrm{CH}_{4}$ ratios are plotted on the $2700-3700 \mathrm{~cm}^{-1}$ wavenumber range on Fig. 5(a).

The intensity maxima of the aliphatic and the amine bands are seen to depend on the $\mathrm{CH}_{4}$ ratio. To further quantify these variations, the amine bands are directly considered, whereas the aliphatic bands are corrected from the 
amine contribution (Fig. 5(b)).

The variation of the two main amine and the three main aliphatic maxima are represented in Fig. 6 as a function of the $\mathrm{CH}_{4}$ ratio. The 'amine' pattern decreases by about a factor of 5 when the $\mathrm{CH}_{4}$ ratio increases and the aliphatic stretching bands of the $\mathrm{CH}_{2}$ and $\mathrm{CH}_{3}$ chemical groups increases by about a factor of 7-8. When the $\mathrm{CH}_{4}$ ratio increases, the material is enriched in aliphatic signatures, whereas the amine content decreases. The absorption in the visible domain may be mainly correlated to the amine absorption bands (Mahjoub et al., 2012). Due to this amine varying content, the imaginary refractive index $k(\lambda)$ in the visible range decreases when the $\mathrm{CH}_{4}$ ratio increases.

Gautier et al. (2012) show that the aliphatic signature at $2900 \mathrm{~cm}^{-1}$ is in general agreement with a feature observed by VIMS (Rannou et al., 2010), and they compare the other bands to the VIMS data.

The absorption by particles in the visible wavelength range, represented numerically by the imaginary refractive index, is mainly due to the chemical composition. The structure of aggregates and the size of the constituent grains influence also the scattered light and its linear polarization.

\section{Light Scattering on Lifted Particles \\ 6.1 PROGRA $^{2}$-vis instrument and experimental method}

The properties (size, refractive indices) studied in the previous sections are parameters which influence the intensity and polarization of the light scattered by the particles. The particles are introduced in a vial and lifted by an air draught. The particles float freely for a few seconds and then progressively fall. This is studied by the PROGRA ${ }^{2}$-vis instrument (a detailed description of the instrument and the procedure is given in Hadamcik et al., 2009b, 2011). The light sources are randomly polarized lasers at $543.5 \mathrm{~nm}$ and $632.8 \mathrm{~nm}$, with a beam diameter of about $2 \mathrm{~mm}$. Optical fibers from the lasers are fixed on the vial, which can rotate to change the phase angle $\alpha$. Two cameras measure the polarized intensities (parallel and perpendicular to the scattering plane). Their resolution corresponds to $20 \mu \mathrm{m}$ at the object. The field of view is about $2 \mathrm{~cm}$. Hundreds of images are used to obtain the polarization at each phase angle, for each pixel or by integration of the polarized intensities on the whole images. The polarization, $P$, is calculated from the difference divided by the sum of the two polarized intensities, respectively perpendicular and parallel to the scattering plane:

$$
P=\frac{I_{\text {perp }}-I_{\text {par }}}{I_{\text {perp }}+I_{\text {par }}}
$$

To build polarization maps, it is necessary to center the images. On the maps the projected surface of the particles is measured and a diameter calculated by the equivalent circle area. Images containing strong multiple scattering effects arising from particles too close to each another are rejected.

The particles in the field of view are aggregates (Hadamcik et al., 2009a). They are generated when the samples are lifted. They are inhomogeneous, made of small aggregates of sintered grains and of individual grains by contact.
The material in the aggregates is sparse. Their size is measured on the polarization maps. The size distribution of the lifted particles can be generally fitted by a Gaussian function. The average size is usually between $50 \mu \mathrm{m}$ and 100 $\mu \mathrm{m}$ with particles between $20 \mu \mathrm{m}$ and $1 \mathrm{~mm}$. If the size distribution of the particles cannot be fitted by a Gaussian function, the polarization results are not considered.

First, $P$ is determined for the whole sample, by integration of the polarized intensities on the whole set of particles and parameters of the phase curves are measured. $P$ can also be studied as a function of the size of the particles in the $20-500 \mu \mathrm{m}$ range. The image of the particles on the camera corresponds to the projection of the field section, with a $\sin (\alpha)$ coefficient. Therefore, the spatial resolution of the particles' images decreases when $\alpha$ decreases. For $\alpha$ $\leq 30^{\circ}$, it becomes impossible to measure the size distribution of the particles. For each size range, and phase angle, the polarized intensities are added and $P$ calculated using Eq. (1). Phase curves are produced for each size range.

\subsection{Polarization phase curves}

Examples of polarization phase curves are given in Fig. 7 at $632.8 \mathrm{~nm}$, and for three $\mathrm{CH}_{4}$ ratios produced under $\mathrm{CWC}$ conditions. They are typical of irregular large particles as compared with the wavelength with a maximum polarization $P_{\max }$ at a phase angle $\alpha_{\max }$ in the range $90^{\circ}-100^{\circ}$, and a negative branch at small phase angles with a minimum polarization $P_{\min }$ at a phase angle $\alpha_{\min }$. The sign of the polarization changes at the so-called inversion angle $\alpha_{0}$. Based on Fig. 7, the differences in the parameters on the phase curves seem to be related to the different $\mathrm{CH}_{4}$ ratios, which are themselves related to the physical properties of the particles, mainly the size of their grains (Section 3). I.e., $P_{\max }$ decreases and $P_{\min }$ is more negative when the constituent grain size increases. For experimental reasons associated with experimental set-up constraints, the minimum polarization $P_{\min }$, if $\alpha_{\min }$ is smaller than $15^{\circ}$, is difficult to measure, and only trends can be deduced from the measurements. The maximum polarization $P_{\max }$ is the more sensitive to the particles' properties and the easier to measure.

\subsection{Influence of the size of the grains on the negative branch}

Figure 8 presents $P_{\min }$ and the inversion angle $\alpha_{0}$ as a function of the average size of the constituent grains for all the tholins produced with $2 \%, 5-6 \%$ and $10 \% \mathrm{CH}_{4}$ ratios for which data are available in the negative branch. For some $\mathrm{CH}_{4}$ ratio conditions, the size distribution is broad (Hadamcik et al., 2009a) and, for example, for the $2 \% \mathrm{CH}_{4}$ ratio under $\mathrm{CWC}$ and mainly for the $10 \% \mathrm{CH}_{4}$ ratio, an important number of grains have diameters of the order of, or larger than, the wavelength, inducing some deeper negative branch (Mie scattering) extending on larger phase angles (also seen in Petrova et al., 2004). A deeper negative branch and an increase of the inversion angle are observed for tholins composed of the largest grains. Moreover, an increase of $n(\lambda)$ and a decrease of $k(\lambda)$ from a $1 \%$ to $10 \%$ $\mathrm{CH}_{4}$ ratio induce also a deeper negative branch. Zubko et al. (2009) found similar results by numerical simulations. The inversion angle $\alpha_{0}$ seems to be limited to values smaller than $30^{\circ}$ for absorbing materials (e.g. $2 \% \mathrm{CH}_{4}$ ratio) and reaches $50^{\circ}$ for more transparent ones (e.g. $10 \% \mathrm{CH}_{4}$ ratio). 


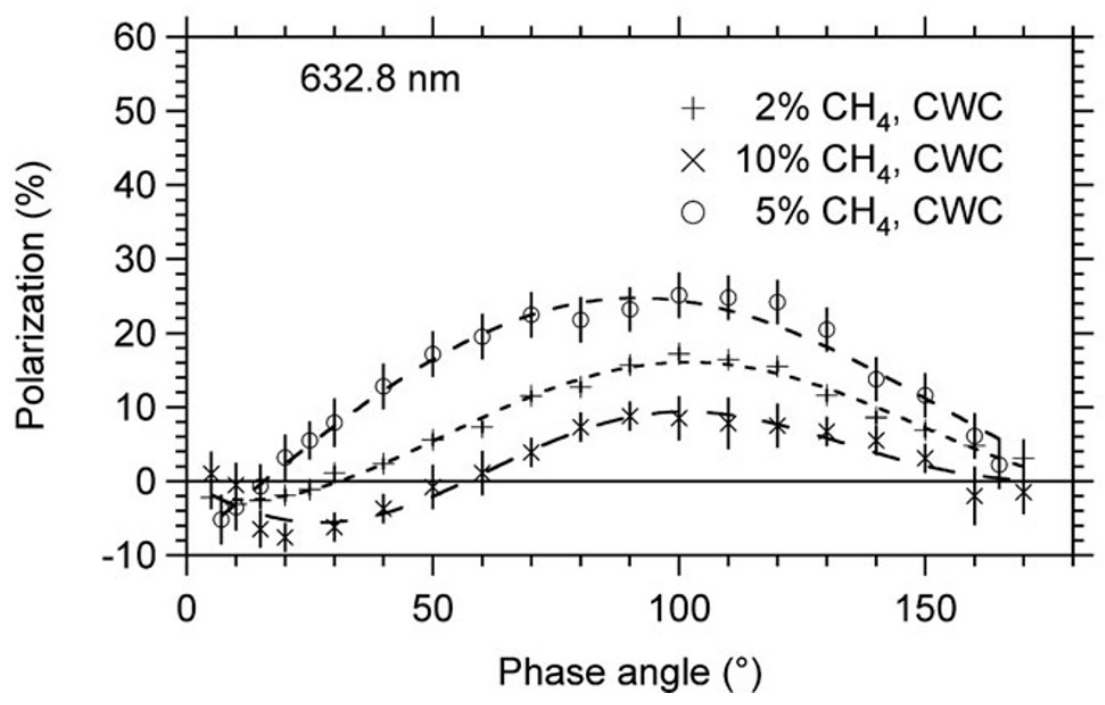

Fig. 7. Polarization vs phase angle for 3 different $\mathrm{CH}_{4}$ ratios.

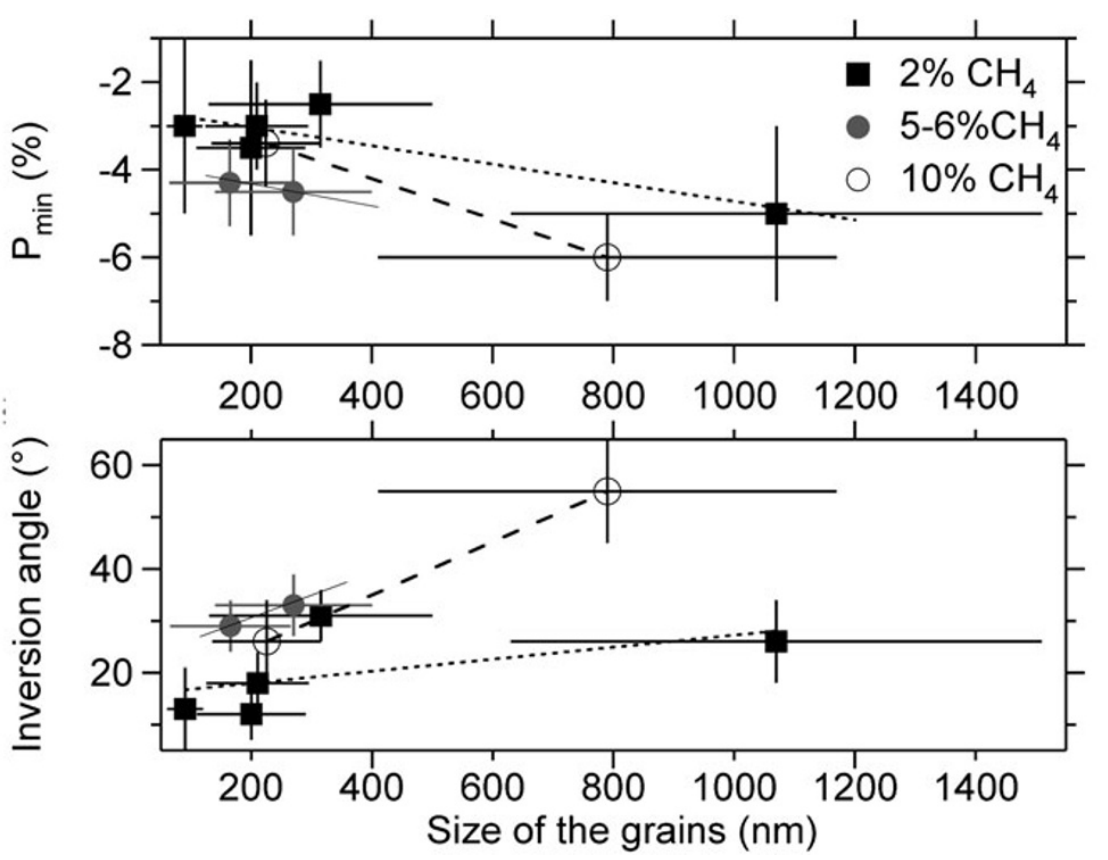

Fig. 8. $P_{\min }$ and $\alpha_{0}$ vs average size of the constituent grains for different $\mathrm{CH}_{4}$ ratios and production conditions. Trends are indicated by a linear fit for each $\mathrm{CH}_{4}$ ratio (5\% and $6 \%$ represented by the same symbols).

These results can be compared with space observations. From outside the atmosphere, the negative polarization on the phase curves is small, with an important scatter of the data (West et al., 1983). From DISR measurements, Tomasko et al. (2009) built a single-scattering polarization phase curve in green light, from a model of fractal cluster-cluster aggregates of 4300 grains with an elemental grain size of 80-nm-diameter mean size. They took into account the radiative transfer in the atmosphere. $P_{\min }$ is about $-2 \%$, which is close to the values found with the experiment for grains with an average diameter smaller than 100 nm (Fig. 8(a)). The inversion angle $\alpha_{0}$ from the Pioneer 11 (Tomasko and Smith, 1982) and DISR/Huygens (Tomasko et al., 2009) seems to be smaller than $10^{\circ}$, which again indicates a grain diameter smaller than $90 \mathrm{~nm}$ (Fig. 8(b)). In order to achieve a more accurate comparison with observations, it is necessary to consider the maximum polarization.

\subsection{Influence of the size of aggregates on the positive} branch

During the experiment, a few seconds after each air draught, the large aggregates fall to the bottom of the vial and are no more in the laser beam. An about uniform faint beam is observed on the screen due to scattering by particles smaller than $10 \mu \mathrm{m}$ (smaller than the camera's resolution). Measurements of the scattered light is possible for a few seconds, and the polarization phase curve can be retrieved. This specific treatment for particles smaller than $10 \mu \mathrm{m}$ is detailed in Hadamcik et al. (2011). $P$ in the positive branch is generally larger for these micrometer-sized aggregates than for the same sample with an average ag- 


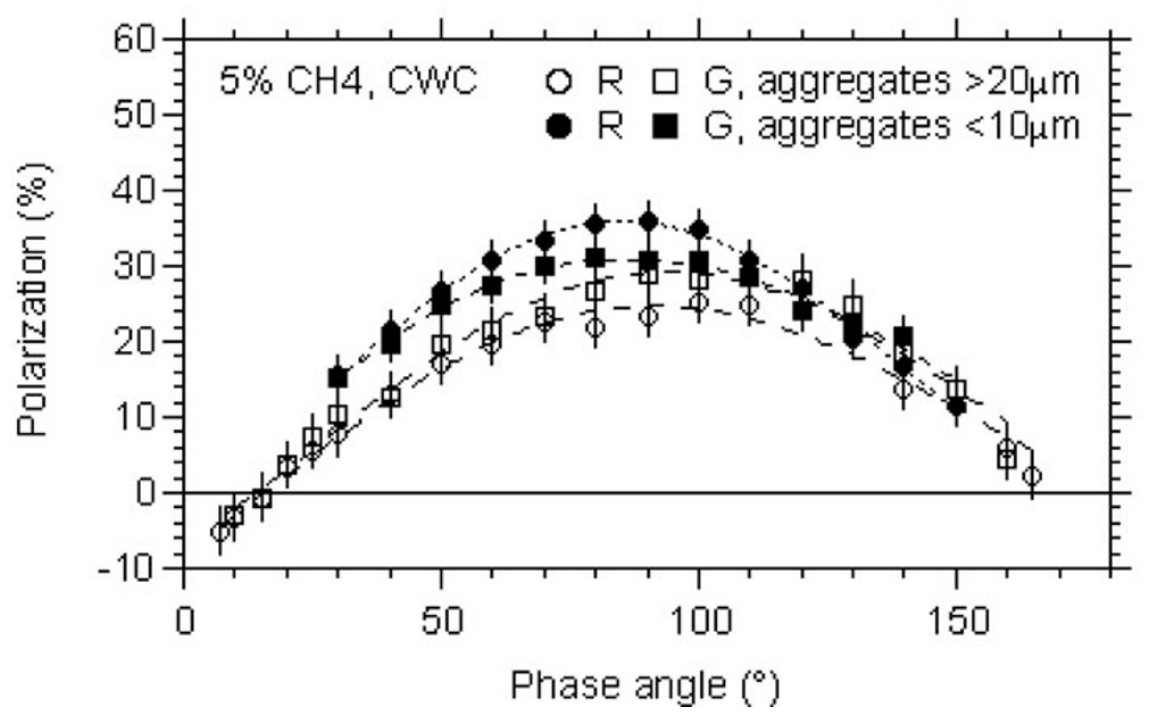

Fig. 9. Comparison of the phase curves for small and large aggregates produced under the same conditions of $5 \% \mathrm{CH}_{4}$.

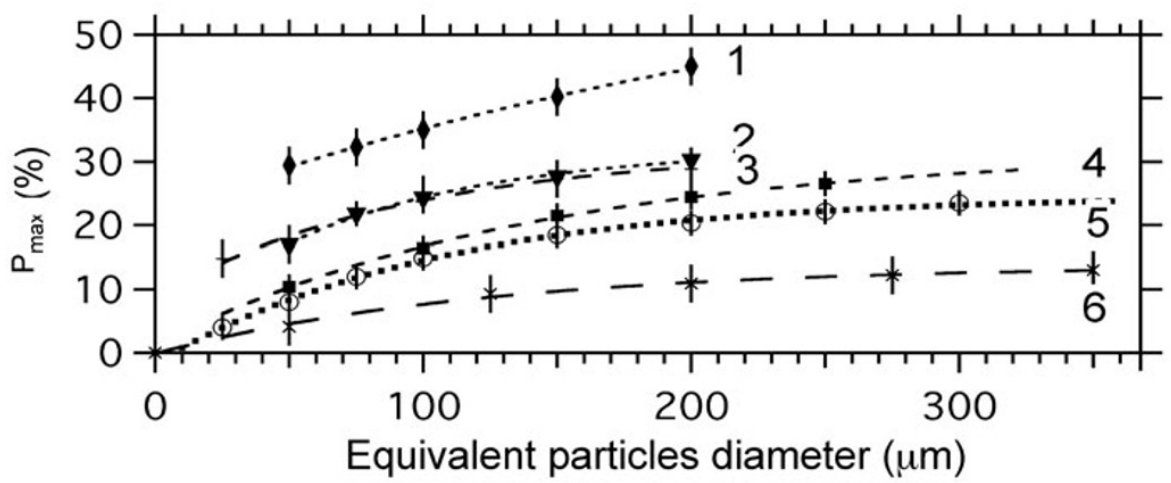

Fig. 10. $P_{\max }$ vs diameter $\geq 20 \mu \mathrm{m}$ of the aggregates (for samples produced at different initial $\mathrm{CH}_{4}$ ratios and plasma durations: (1) $2 \%, 10 \mathrm{~s}$; (2) $2 \%$, $50 \mathrm{~s}$; (3) $10 \%, 40 \mathrm{~s}$; (4) $5 \%$, CWC; (5) $2 \%$, CWC; (6) $10 \%$, CWC.

gregate size between $50 \mu \mathrm{m}$ and $100 \mu \mathrm{m}$ (Hadamcik et al., 2009a) as, for example, for the phase curves obtained for the $5 \% \mathrm{CH}_{4}$ ratio sample presented in Fig. 9 (discussed on the wavelength influence in Subsection 6.6).

The polarization is calculated for each size range (diameter $\geq 20 \mu \mathrm{m}$ ) of the aggregates and the variation of $P_{\max }$ is presented in Fig. 10 for red light. When the size of the particles increases, $P_{\max }$ increases up to a maximum for all the samples.

\subsection{Influence of the size of the grains on $\boldsymbol{P}_{\max }$}

The size of the constituent grains is also an important parameter. As was seen in Section 3, the size of the grains depends on the plasma duration in the pulsed mode, and on the $\mathrm{CH}_{4}$ ratio in the $\mathrm{CWC}$ mode. The maximum polarization $P_{\max }$ increases when the grain size decreases for the two wavelengths (Fig. 11). It was first studied for the $2 \% \mathrm{CH}_{4}$ ratio at the same wavelength with different plasma durations (Fig. 11, black symbols) and for all the tholins produced under various conditions (open symbols). As was seen in Section 4, the refractive indices depend on the $\mathrm{CH}_{4}$ ratios, but their influence on the maximum polarization seems to be small compared with the influence of the equivalent diameter of the grains (smaller than, or about equal to the wavelength).

If the polarization values close to maximum, as deduced from the observations made onboard the space probes, are extrapolated to the PROGRA ${ }^{2}$ experiment wavelength values, the average equivalent diameter of the constituent grains have to be about $80 \mathrm{~nm}$ (Fig. 11 and Table 1).

\subsection{Influence of the wavelength on $\boldsymbol{P}_{\max }$}

The positive polarization and its maximum $P_{\max }$ change with a change of the incident wavelength (Figs. 9 and 11). For large aggregates, the positive polarization decreases when the wavelength increases. For aggregates with a diameter smaller than $10 \mu \mathrm{m}$, the opposite behavior occurs with an increase of the positive polarization.

The size parameter depends on $d / \lambda$ : an increase of $\lambda$ is equivalent to a decrease of the size. The refractive indices are also dependent on the wavelength (Section 4). These different parameters will be discussed to disentangle their respective influence. When $\lambda$ decreases from $632.8 \mathrm{~nm}$ to $543.5 \mathrm{~nm}$, it is equivalent to multiplying the sizes by about 1.16. When the size parameter of the large aggregates increases, $P_{\max }$ increases (Fig. 10) by $1.5 \%$ on average for sizes in the 50-100 $\mu \mathrm{m}$ range. When the size parameter of the grains increases, $P_{\max }$ decreases (Fig. 11), the varia- 


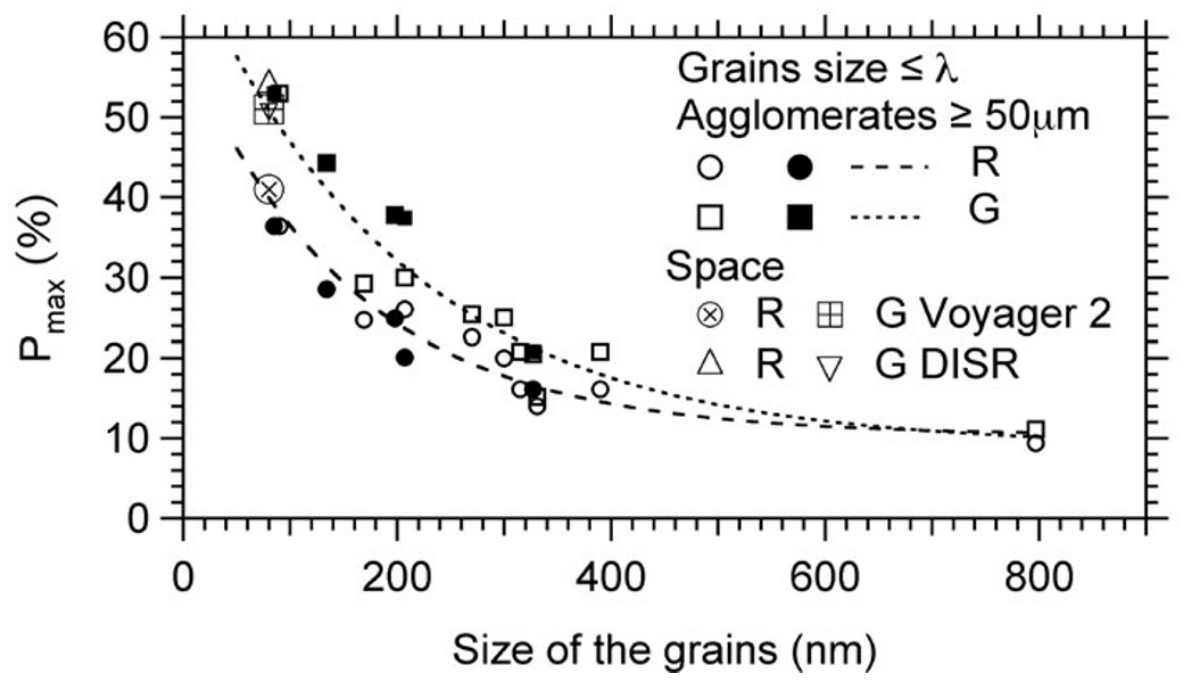

Fig. 11. $P_{\max }$ vs size of the grains for a size distribution of the aggregates with an average between $50 \mu \mathrm{m}$ and $100 \mu \mathrm{m}$ in two wavelengths. Black symbols for $2 \% \mathrm{CH}_{4}$, open symbols for all other ratios. Fits are for all the ratios. Space results extrapolated to $R=632.8 \mathrm{~nm}$ and $G=543.5 \mathrm{~nm}$ for a supposed grain size of $80 \mathrm{~nm}$ for comparison. Updated from Hadamcik et al. (2009a).

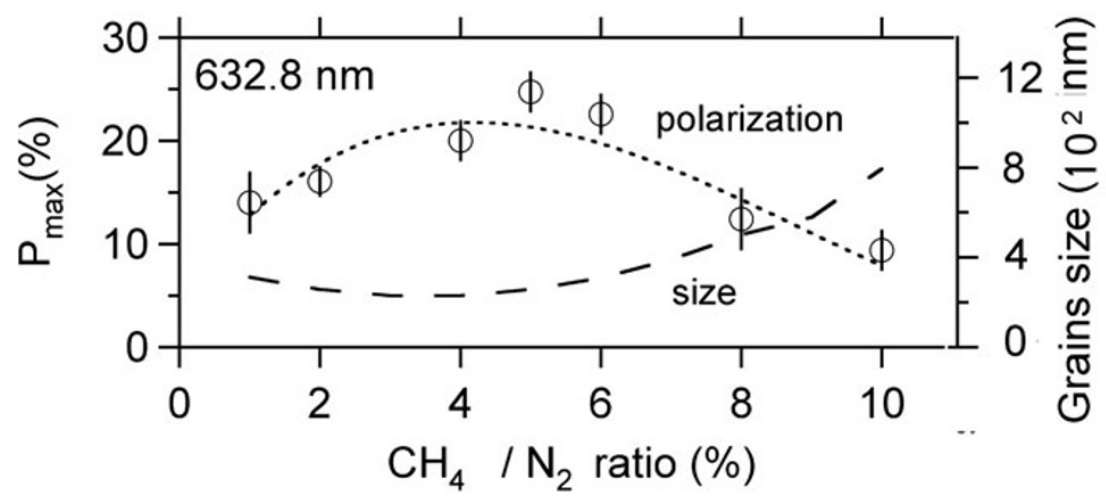

Fig. 12. $P_{\max }$ (left vertical axis) and average grain size (right axis) vs $\mathrm{CH}_{4}$ initial.

tion is smaller for large grains $(1.4 \%$ on average for grains with a $80-\mathrm{nm}$ diameter to $1.1 \%$ for grains with a $400-\mathrm{nm}$ diameter). As a consequence, $P_{\max }$ increases slightly, or is constant, when the wavelength decreases for large aggregates. For small aggregates (micrometer sized), numerical simulations suggest an increase of $P_{\max }$ when the aggregate and constituent grain sizes decrease, if $n$ and $k$ are in the range found for tholins (Petrova et al., 2004). As a consequence, the decrease of the size parameter from green to red can explain the increase of positive polarization for aggregates smaller than $10 \mu \mathrm{m}$ with the increase of wavelength. This behavior is different for larger aggregates (Fig. 9).

The polarization depends also on the refractive indices. When $\lambda$ decreases, $n(\lambda)$ and $k(\lambda)$ increases. Numerical models are limited in the size of the particles to equivalent diameters of some micrometers: they are used in the present paper to give trends. When $n(\lambda)$ increases from 1.5 to 1.9, $P_{\text {max }}$ decreases for aggregates with an equivalent diameter smaller than about $10 \mu \mathrm{m}$ (Petrova et al., 2004) and a constant absorption $k$. For a constant value of $n(\lambda)$, when $k(\lambda)$ increases, $P_{\max }$ increases. Zubko et al. (2009) aggregates' size parameter is in the 5 to 30 range (equivalent particle size in the 1 to $6 \mu \mathrm{m}$ range), they consider the variation of
Table 1. Comparison between laboratory and space measurements (extrapolated to $R=632.8 \mathrm{~nm}$ and $G=543.5 \mathrm{~nm}$ ). The average diameter of the grains in the aggregates is $75 \mathrm{~nm}$. Values updated from Hadamcik et al. (2009a). Measurements for the small aggregates with $2 \% \mathrm{CH}_{4}$.

\begin{tabular}{lcc}
\hline & $P_{\max }(G) \%$ & $P_{\max }(R) \%$ \\
\hline Laboratory & & \\
Aggregates $\geq 20 \mu \mathrm{m}$ & $53 \pm 2$ & $38 \pm 2$ \\
Aggregates $\leq 10 \mu \mathrm{m}$ & $54 \pm 3$ & $55 \pm 3$ \\
\hline Space observations & & \\
Integrated from outside & $\approx 49$ & $\approx 41$ \\
Upward from inside (DISR) & $\approx 51$ & $\approx 54$ \\
\hline
\end{tabular}

$P_{\max }$ as a function of ' $k$ ' with a fixed $n$ at 1.5 . For $k \leq 0.05$, corresponding to tholins, the increase of $P_{\max }$ with increasing $k$ is very important for the largest aggregates. In that case, $P_{\max }(543.5)$ is higher than $P_{\max }(632.8)$, as observed for the tholins for large aggregates, but it keeps the same value or slightly decreases for the smallest ones. The variation of $P_{\max }$ seems to be dominated by the absorption for the large aggregates.

Internal interactions depolarize the light, as is well observed for transparent particles with small $k(\lambda)$ values 
(Hadamcik et al., 2009c); it is also found in numerical simulations on small aggregates (Kimura et al., 2003; Petrova et al., 2004). As the size of the aggregate increases, more light is absorbed. For sufficiently large relatively absorbing particles, the emergent light is mainly scattered by the external grains and the polarization has a maximum value due to the absence of depolarization by multiple scattering, e.g. in green light (Hadamcik et al., 2009a).

Similar conclusions can be deduced for the grains' size from the PROGRA ${ }^{2}$ experiment and from the space observations (Tomasko et al., 2009). The polarization values obtained for the green light is about the same for large and small aggregates. They are similar when measured from outside the atmosphere by integrating the fluxes (mainly sensitive to particles in the upper atmosphere) and from inside the atmosphere by the DISR/Huygens experiment looking upward (Table 1). In red light, the maximum polarization values are smaller for large aggregates than for small ones (more internal interactions due to the smaller absorption). Similarly, the external observations of Titan's atmosphere from Pioneer 11 and Voyager 2 space probes give smaller values because of multiple scattering in the atmosphere, compared with measurements carried out inside the atmosphere looking upward by the DISR, when extrapolated to the wavelength used in the experiment. The integration over the whole atmosphere increases the depolarization by multiple scattering for larger wavelengths (Hadamcik et al., 2009a).

The influence of the wavelength on the maximum polarization is an important factor to consider for a comparison with space observations. However, the quantitative effect on $P_{\max }$ is small as compared with the grain size influence (Fig. 11).

\subsection{Conclusion on the influence of the $\mathrm{CH}_{4}$ ratio}

The minimum polarization, $P_{\min }$, the maximum polarization, $P_{\max }$, and the inversion angle, $\alpha_{0}$, for the different samples have been found to mainly depend on the size of the constituent grains. However, the samples produced with various methane ratios show different average grain sizes (Fig. 3).

In Fig. 12, the variation of $P_{\max }$ is presented as a function of the $\mathrm{CH}_{4}$ ratio, such as the variation of the average grain sizes. A maximum value of $P_{\max }$ is observed for ratios between $4 \%$ and $6 \% \mathrm{CH}_{4}$. And, indeed, the behavior of the positive polarization is anti-correlated to the variation of the size of the grains as a function of the $\mathrm{CH}_{4}$ ratio.

\section{Summary and Conclusion}

Tholins optical properties have been studied emphasizing their variation as a function of the $\mathrm{CH}_{4} / \mathrm{N}_{2}$ ratio with the PAMPRE set-up. SEM analysis has enabled the constituent grain-size distribution to be measured for each sample. A minimum size is obtained for $\mathrm{CH}_{4} / \mathrm{N}_{2}$ ratios between $4 \%$ and $5 \%$. The linear polarization phase curves are dominated by the variation in the size of the constituent grains. An anti-correlation is observed between the grain-size variation and the maximum polarization, $P_{\max }$. The negative branch is also dependent on the $\mathrm{CH}_{4} / \mathrm{N}_{2}$ ratio by the grain-size dependence. An initial investigation has been conducted and future investigations are needed for low phase angles be- cause of the critical signal-to-noise ratio values in this region. $P_{\min }, \alpha_{0}$ and $P_{\max }$ values for grain sizes smaller than, or equal to, 90 -nm diameters are similar to those found by the different space missions (when the values are extrapolated to the same wavelengths). The large aggregates values correspond to values integrated over the whole satellite atmosphere, as measured by Pioneer 11 and Voyager 2, and the values observed by DISR/Huygens looking upward to values obtained for aggregates in the micrometer-size range.

Acknowledgments. EH would like to thank H. Kimura and the CPS for organizing the Cosmic Dust workshop and for their help, allowing her to participate. The authors thank S. Borensztajn (Univ. Paris-Sorbonne, UPR 15) for the SEM-FEG images. CNES is acknowledged for funding the PROGRA ${ }^{2}$ experiment. Financial support for A. M. post-doc (ANR-09-JCJC-0038 contract).

\section{References}

Gautier, T., N. Carrasco, A. Mahjoub, S. Vinatier, A. Giufiani, C. Szopa, C. M. Anderson, J. J. Correia, P. Dumas, and G. Cernogora, Mid- and farinfrared absorption spectroscopy of Titan's aerosols analogues, Icarus, 220, 320-327, 2012.

Hadamcik, E., J.-B. Renard, G. Alcouffe, G. Cernogora, A. C. LevasseurRegourd, and C. Szopa, Laboratory light-scattering measurements with Titan's aerosols analogues produced by a dusty plasma, Planet. Space Sci., 57, 1631-1641, 2009a.

Hadamcik, E., J.-B. Renard, A. C. Levasseur-Regourd, and J. C. Worms, Laboratory measurements of the light scattered by clouds of solid particles by imaging technique, in Light Scattering Rev 4, Single Light Scattering and Radiative Transfer, edited by A. Kokhanovsky, 40 pp., Springer-Praxis, Berlin, 2009b.

Hadamcik, E., J.-B. Renard, A. C. Levasseur-Regourd, J. Lasue, G. Alcouffe, and M. Francis, Light scattering by agglomerates: Interconnecting size and absorption effects (PROGRA ${ }^{2}$ experiment), J. Quant. Spectrosc. Radiat. Transfer, 110, 1755-1770, 2009c.

Hadamcik, E., J.-B. Renard, A. C. Levasseur-Regourd, and J. Lasue, Laboratory measurements of light scattered by clouds and layers of solid particles using an imaging technique, in Proceedings of the NATO Advanced Study Institute on Special Detection Technique (Polarimetry) and Remote Sensing, edited by M. I. Mishchenko, Y. S. Yatskiv, V. K. Rosenbush, and G. Videen, 40 pp., NATO Science for Peace and Security Series C: Environmental Security, Springer, Yalta, Ukraine 20 September-1 October 2010, 2011.

Imanaka, H., B. N. Khare, J. E. Elsila, E. L. O. Bakes, C. P. McKay, D. P. Cruiskshank, S. Sugita, T. Matsui, and R. N. Zare, Laboratory experiments of Titan tholin formed in cold plasma at various pressures: Implications for nitrogen-containing polycyclic aromatics compounds in Titan haze, Icarus, 168, 344-366, 2004.

Khare, B. N., K. Sagan, E. T. Arakawa, F. Suits, T. A. Callcott, and M. W. Williams, Optical constants of organic tholins produced in a simulated Titanian atmosphere: From soft X-ray to microwave frequencies, Icarus, 60, 127-137, 1984.

Kimura, H., L. Kolokolova, and I. Mann, Optical properties of cometary dust. Constraints from numerical studies on light scattering by aggregate particles, Astron. Astrophys., 407, L5-L8, 2003.

Mahjoub, A., N. Carrasco, P.-R. Dahoo, T. Gautier, C. Szopa, and G. Cernogora, Influence of methane concentration on the optical indices of Titan's aerosols analogues, Icarus, 221, 670-677, 2012.

Petrova, E., V. P. Tishkovets, and K. Jockers, Polarization of light scattered by solar system bodies and the aggregate model of dust particles, Sol. Syst. Res., 38, 309-324, 2004.

Quirico, E., G. Montagnac, V. Lees, P. F. McMillan, C. Szopa, G. Cernogora, J. N. Rouzaud, P. Simon, J. M. Bernard, P. Coll, N. Fray, R. D. Minard, F. Raulin, B. Reynard, and B. Schmitt, New experimental constraints on the composition and structure of tholins, Icarus, 198, 218-231, 2008.

Rannou, P., T. Cours, S. Le Mouélic, S. Rodriguez, C. Sotin, P. Drossart, and R. Brown, Titan haze distribution and optical properties retrieved from recent observations, Icarus, 208, 850-867, 2010.

Renard, J.-B., J. C. Worms, T. Lemaire, E. Hadamcik, and N. Huret, Lifght scattering by dust particles in microgravity: Polarization and brightness imaging with the new version of the PROGRA ${ }^{2}$ instrument, Appl. Opt., 
41, 609-618, 2002.

Sciamma-O’Brien, E., N. Carrasco, C. Szopa, A. Buch, and G. Cernogora, Titan's atmosphere: An optimal gas mixture for aerosol production?, Icarus, 209, 704-714, 2010.

Sciamma-O’Brien, E., P.-R. Dahoo, E. Hadamcik, N. Carrasco, E. Quirico, C. Szopa, and G. Cernogora, Optical constants from $370 \mathrm{~nm}$ to $900 \mathrm{~nm}$ of tholins produced in a low pressure RF plasma discharge, Icarus, $\mathbf{2 1 8}$, 356-363, 2012.

Szopa, C., G. Cernogora, L. Boufendi, J. J. Correia, and P. Coll, PAMPRE a dusty plasma experiment for Titan's tholins production and study, Planet. Space Sci., 54, 394-404, 2006.

Tomasko, M. G. and P. H. Smith, Photometry and polarimetry of Titan: Pioneer 11 observations and their implications for aerosol properties, Icarus, 51, 65-95, 1982.

Tomasko, M. G., B. Archinal, T. Becker, B. Bézard, M. Bushroe, M. Combes, D. Cook, A. Coustenis, C. de Bergh, and 30 coauthors, Rain, winds and haze during the Huygens probe's descent to Titan's surface, Nature, 438, 765-778, 2005.
Tomasko, M. G., L. Doose, S. Engel, L. E. Dafoe, R. West, and M. Lemmon, A model of Titan's aerosols based on measurements made inside the atmosphere, Planet. Space Sci., 56, 669-707, 2008.

Tomasko, M. G., L. Doose, L. E. Dafoe, and C. See, Limits on the size of aerosols from measurements of linear polarization in Titan's atmosphere, Icarus, 204, 271-283, 2009.

West, R. A., H. Hart, K. E. Simmons, C. W. Hord, L. W. Esposito, A. L. Lane, R. B. Pomphrey, D. L. Coffeen, and M. Sato, Voyager 2 photopolarimeter observations of Titan, J. Geophys. Res., 88, A118699A118708, 1983.

Zubko, E., H. Kimura, Y. Shkuratov, K. Muinonen, T. Yamamoto, H Okamoto, and G. Videen, Effect of absorption on light scattering by agglomerated debris particles, J. Quant. Spectrosc. Radiat. Transfer, 110, 1741-1749, 2009.

E. Hadamcik (e-mail: edith.hadamcik@latmos.ipsl.fr), J.-B. Renard, A. Mahjoub, T. Gautier, N. Carrasco, G. Cernogora, and C. Szopa 